\title{
Effects of Conversational Agents on Human Communication in Thought-Evoking Multi-Party Dialogues
}

\author{
Kohji Dohsaka \\ NTT Communication Science Laboratories \\ NTT Corporation \\ Ryota Asai \\ Graduate School of \\ 2-4, Hikaridai, Seika-cho, \\ Kyoto 619-0237, Japan \\ Information Science and Technology \\ Osaka University, 1-1 Yamadaoka, \\ Suita, Osaka 565-0871, Japan \\ Ryuichiro Higashinaka and Yasuhiro Minami and Eisaku Maeda \\ NTT Communication Science Laboratories, NTT Corporation \\ 2-4, Hikaridai, Seika-cho, Kyoto 619-0237, Japan \\ \{dohsaka, rh, minami, maeda\}@cslab.kecl.ntt.co.jp
}

\begin{abstract}
This paper presents an experimental study that analyzes how conversational agents activate human communication in thought-evoking multi-party dialogues between multi-users and multi-agents. A thought-evoking dialogue, which is a kind of interaction in which agents act on user willingness to provoke user thinking, has the potential to stimulate multi-party interaction. In this paper, we focus on quiz-style multi-party dialogues between two users and two agents as an example of a thought-evoking multi-party dialogue. The experiment results showed that the presence of a peer agent significantly improved user satisfaction and increased the number of user utterances. We also found that agent empathic expressions significantly improved user satisfaction, raised user ratings of a peer agent, and increased user utterances. Our findings will be useful for stimulating multi-party communication in various applications such as educational agents and community facilitators.
\end{abstract}

\section{Introduction}

Conversational interfaces including dialogue systems and conversational agents have been typically used as a single interface to a single user (Zue et al., 1994; Allen et al., 2001; Cassell et al., 2000). On the other hand, a new area of research in conversational interfaces is dealing with multi-party interaction (Traum and Rickel, 2002; Liu and Chee, 2004; Zheng et al., 2005). Multiparty conversational interfaces have been applied to such tasks as training decision-making in team activities (Traum and Rickel, 2002), collaborative learning (Liu and Chee, 2004), and coordinating and facilitating interaction in a casual social group (Zheng et al., 2005).

The advantage of such multi-party dialogues over two-party cases is that the multi-party case encourages group interaction and collaboration among human users. This advantage can be exploited to foster such human activities as student learning in more social settings and to build and maintain social relationships among people. However, unless users actively engage in the interaction, these multi-party dialogue qualities cannot be adequately exploited. Our objective is to stimulate human communication in multi-party dialogues between multi-users and multi-agents by raising user willingness to engage in the interaction and increasing the number of user utterances.

As the first step toward this objective, we exploit a new style of dialogue called thoughtevoking dialogue and experimentally investigate the impact of a peer agent's presence and agent emotional expressions on communication activation in thought-evoking multi-party dialogues. A thought-evoking dialogue, an interaction in which agents act on the willingness of users to provoke user thinking and encourage involvement in the dialogue, has the potential to activate interaction among participants in multi-party dialogues.

Previous work proposed a quiz-style information presentation dialogue system (hereafter quizstyle dialogue system) (Higashinaka et al., 2007a) that is regarded as a kind of thought-evoking dialogue system. This system conveys contents as biographical facts of famous people through quizstyle interaction with users by creating a "Who is this?" quiz and individually presenting hints. 
The hints are automatically created from the biographical facts of people and ordered based on the difficulty naming the people experienced by the users (Higashinaka et al., 2007b). Since the user has to consider the hints to come up with reasonable answers, the system stimulates user thinking. This previous work reported that, for interaction between a single user and a computer, a quiz-style dialogue improved user understanding and willingness to engage in the interaction. In this paper, we focus on a quiz-style information presentation multi-party dialogue (hereafter quiz-style multi-party dialogue) as an example of a thought-evoking multi-party dialogue.

A peer agent acts as a peer of the users and participates in the interactions in the same way that the users do. We are interested in the peer agent's role in quiz-style multi-party dialogues since the positive effects of a peer agent on users have been shown in the educational domain (Chou et al., 2003; Maldonado et al., 2005), which is a promising application area for quiz-style dialogues. In the educational domain, a user could benefit not only from direct communication with a peer agent but also from overhearing dialogues between a peer agent and a tutor. Learning by observing others who are learning is called vicarious learning and positively affects user performance (Craig et al., 2000; Stenning et al., 1999). To the best of our knowledge, detailed experimental investigations on the effect of a peer agent on communication activation have not been reported in multi-party dialogues between multi-users and multi-agents, which are our main concern in this paper.

The topic of emotion has gained widespread attention in human-computer interaction (Bates, 1994; Picard, 1997; Hudlicka, 2003; Prendinger and Ishizuka, 2004). The impact of an agent's emotional behaviors on users has also recently been studied (Brave et al., 2005; Maldonado et al., 2005; Prendinger et al., 2005). However, these previous studies addressed scenario-based interaction in which a user and an agent acted with predetermined timing. In this paper, we investigate the impact of agent emotional expressions on users in multi-party dialogues in which multiple users and agents can make utterances with more flexible timing.

Resembling work by Brave et al. (2005), we classify agent emotional expressions into empathic and self-oriented ones and investigate their impact on users in a thought-evoking multi-party dialogue system. As stated above, Brave et al. (2005) addressed scenario-based Black-jack interaction, but we deal with multi-party dialogues that enable more flexible turn-taking. Previous studies (Bickmore and Picard, 2005; Higashinaka et al., 2008) showed that agent empathic expressions have a positive psychological impact upon users, but they only examined two-party cases. Although Traum et al. (2002) and Gebhard et al. (2004) exploited the role of agent emotion in multi-party dialogues, they did not adequately examine the effects of agent emotion on communication activation by experiment.

In this work, we deal with disembodied agents and focus on their linguistic behaviors. We believe that our results are useful for designing embodied conversational agents using other modalities.

This paper presents an experimental study that analyzes how agents stimulate human communication in quiz-style multi-party dialogues between two users and two agents. We are especially interested in how the presence of a peer agent and agent emotional expressions improve user satisfaction, enhance user opinions about the peer agent, and increase the number of user utterances. Our findings will be useful for stimulating human communication in various applications such as educational agents and community facilitators.

In the following, Section 2 shows an overview of our quiz-style multi-party dialogue system. Section 3 explains the experiment design, and Section 4 describes the results. Section 5 concludes the paper.

\section{Thought-Evoking Multi-Party Dialogue System}

We implemented a quiz-style multi-party dialogue system between multi-users and multi-agents. The system is a Japanese keyboard-based dialogue system with a chat-like interface. The users can make utterances any time they want. A user utterance is completed and displayed on the chat window when the Enter key is pressed.

Our experiment dealt with cases where two users and two agents engaged in a dialogue. The two agents are a quizmaster and a peer. The quizmaster agent creates a "Who is this?" quiz about a famous person and presents hints one by one to the users and the peer agent who guess the correct answer. 
1 Whowho Who is this? First hint: Graduated from the University of Tokyo.

2 Mary I don't know.

3 Kinoko Yoshida Shigeru.

4 Whowho No, not even close!

5 Jane That's very difficult.

6 Kinoko Difficult for me, too.

7 Whowho Second hint: Born in Ushigome, Edo.

8 Whowho Third hint: Novelist and scholar of British literature.

9 Mary Murakami Haruki.

10 Whowho Close!

11 Kinoko You are close. Excellent.

12 Jane Well then, who is it?

13 Whowho Fourth hint: Familiar with Haiku, Chinese poetry, and calligraphy.

14 Mary Natsume Soseki.

15 Whowho That's right. Wonderful.

16 Kinoko Mary, excellent. I'm happy for you.

17 Jane Mary, that's the right answer. Good job.

Figure 1: Sample dialogue

Figure 1 shows a sample dialogue. Mary and Jane are human users. Whowho is the quizmaster agent, and Kinoko is the peer agent. Quizmaster agent Whowho presents hints in lines 1, 7, 8, and 13. Users Mary and Jane and peer agent Kinoko give answers in lines 3, 9, and 14 .

The hints were automatically created using biographical facts (in Japanese) of people in Wikipedia ${ }^{1}$ based on a previously reported method (Higashinaka et al., 2007b).

\subsection{Dialogue acts}

The users and the two agents perform several dialogue acts based on the dialogue context.

Present-hint: The quizmaster agent presents hints one by one (lines 1, 7, 8, and 13) in the sample dialogue shown in Figure 1.

Give-ans: Users and the peer agent give answers (lines 3, 9, and 14).

Show-difficulty: Users and the peer agent offer opinions about the quiz difficulty (lines 2, 5, 6, and 12).

\footnotetext{
${ }^{1}$ http://ja.wikipedia.org/
}

Evaluate-ans: When the answer is wrong, the quizmaster agent evaluates the answer based on the person-name similarity score (Higashinaka et al., 2007a) and utters "very close!," "close!," "a little close!," "a little far," "far," or "not even close!" (lines 4 and 10).

Complete-quiz-with-success: When the right answer is given, the quizmaster agent informs the dialogue participants that the current quiz is completed (line 15).

Complete-quiz-with-failure: If all hints have been generated and no right answer is given, the quizmaster agent gives the right answer, and the current quiz is completed.

Feedback-on-wrong-ans: Users and the peer agent give feedback when their own or the other's answers are wrong during the current quiz (line 11).

Feedback-on-success: Users and the peer agent give feedback when their own or the other's answers are right and the current quiz session is completed (lines 16 and 17).

Feedback-on-failure: Users and the peer agent give feedback when the current quiz is completed without the right answer.

Address-hearer: Users and the two agents specify an intended addressee by uttering the other's name (lines 16 and 17).

When a user utterance is input, the system separates it into word tokens using a Japanese morphological analyzer and converts it into dialogue acts using hand-crafted grammar. The system can recognize 120,000 proper names of persons.

\subsection{Utterance generation}

Surface realization forms were prepared for each dialogue act by the agents. Agent utterances are generated by randomly selecting one of the forms.

Some agent dialogue acts can be generated with emotional expressions. Agent emotional expressions are categorized into empathic and selforiented ones (Brave et al., 2005). The agent self-oriented emotional expressions (self-oriented expressions) are oriented to their own state, and the agent empathic expressions are oriented to the other's state and are congruent with the other's 


\begin{tabular}{|l|c|l|}
\hline Dialog act & Emotion & Expressions \\
\hline \hline $\begin{array}{l}\text { Show- } \\
\text { difficulty }\end{array}$ & EMP & Difficult for me, too. \\
\hline $\begin{array}{l}\text { Show- } \\
\text { difficulty }\end{array}$ & SELF & $\begin{array}{l}\text { I don't remember. } \\
\text { That's so frustrating. }\end{array}$ \\
\hline $\begin{array}{l}\text { Show- } \\
\text { difficulty }\end{array}$ & NONE & I don't know. \\
\hline $\begin{array}{l}\text { Feedback- } \\
\text { on-success }\end{array}$ & EMP & $\begin{array}{l}\text { You're right. I'm } \\
\text { happy for you. }\end{array}$ \\
\hline $\begin{array}{l}\text { Feedback- } \\
\text { on-success }\end{array}$ & SELF & $\begin{array}{l}\text { I'm really glad I got } \\
\text { the correct answer. }\end{array}$ \\
\hline $\begin{array}{l}\text { Feedback- } \\
\text { on-success }\end{array}$ & NONE & $\begin{array}{l}\text { You're right / I'm } \\
\text { right. }\end{array}$ \\
\hline $\begin{array}{l}\text { Feedback- } \\
\text { on-failure }\end{array}$ & EMP & $\begin{array}{l}\text { Too bad you didn't } \\
\text { know the right an- } \\
\text { swer. }\end{array}$ \\
\hline $\begin{array}{l}\text { Feedback- } \\
\text { on-failure }\end{array}$ & SELF & $\begin{array}{l}\text { I'm disappointed } \\
\text { that I didn't know } \\
\text { the right answer. }\end{array}$ \\
\hline $\begin{array}{l}\text { Feedback- } \\
\text { on-failure }\end{array}$ & NONE & $\begin{array}{l}\text { I/You didn't know } \\
\text { the right answer. }\end{array}$ \\
\hline
\end{tabular}

Table 1: Examples of agent expressions. EMP shows empathic expressions, SELF shows selforiented expressions, and NONE shows neutral expressions when neither emotion is present.

welfare. As explained in 3.1, we prepared different experimental conditions to determine the presence/absence of agent empathic and self-oriented expressions. Based on the conditions, we controlled the agent emotional expressions. Table 1 shows examples of agent empathic, self-oriented, and neutral expressions.

\subsection{Dialogue management}

The system maintains a dialogue state in which the history of the participant's dialogue acts is recorded with the time of each act. We prepared preconditions of each dialogue act by the agents. For example, the quizmaster agent's Evaluateans can be executed after the users or the peer agent provides a wrong answer. The peer agent's Feedback-on-success can be executed after the quizmaster agent performs Complete-quiz-withsuccess. We also used the following turn-taking rules:

1. Either agent must talk when neither the users nor the agents make utterances within a given time (4 sec.).

\begin{tabular}{|c|l|l|l|}
\hline Condition & $\begin{array}{l}\text { Peer } \\
\text { agent }\end{array}$ & Empathic & $\begin{array}{l}\text { Self- } \\
\text { oriented }\end{array}$ \\
\hline$(0)$ & Absent & Absent & Absent \\
$(1)$ & Present & Absent & Absent \\
$(2)$ & Present & Present & Absent \\
$(3)$ & Present & Absent & Present \\
$(4)$ & Present & Present & Present \\
\hline
\end{tabular}

Table 2: Experimental conditions based on presence/absence of peer agent and agent empathic and self-oriented expressions

2. Agents must not talk for a given time $(0.5$ sec.) after the others talk.

3. The quizmaster agent must move to the next hint when neither the users nor the peer agent give a correct answer within a given time (30 sec.).

Based on the dialogue state, the preconditions of the dialogue acts and the turn-taking rules, the system chooses the next speaker and its dialogue act.

\section{Experiment}

\subsection{Experimental conditions}

To evaluate the effects of the presence of the peer agent and the agent emotional expressions, we prepared five systems under different experimental conditions, (0), (1), (2), (3), and (4), based on the presence/absence of the peer agent and agent empathic and self-oriented expressions. They are shown in Table 2. In condition (0), the peer agent was absent, and only the quizmaster agent was present. In other conditions, both the quizmaster and peer agents were present. In conditions (0) and (1), neither empathic nor self-oriented expressions were exhibited. In condition (2), only empathic expressions were exhibited. In condition (3), only self-oriented expressions were exhibited. In condition (4), both empathic and self-oriented expressions were exhibited.

We evaluated the effects of the presence of the peer agent by comparing conditions (0) and (1). We evaluated the effects of agent empathic and self-oriented expressions by comparing conditions (1), (2), (3), and (4).

\subsection{Measures}

We used three measures: user satisfaction, user opinions about the peer agent, and the number of 


\begin{tabular}{|l|l|}
\hline & Questionnaire items \\
\hline \hline Q1 & $\begin{array}{l}\text { Did you want to converse with this sys- } \\
\text { tem again? (Willingness to engage in di- } \\
\text { alogue) }\end{array}$ \\
Q3 & $\begin{array}{l}\text { Was the dialogue enjoyable? (Pleasant- } \\
\text { ness of dialogue) } \\
\text { Did you feel satisfied using the dialogue } \\
\text { system? (Satisfaction of system usage) } \\
\text { Qas the peer agent friendly? (Agent's } \\
\text { closeness) }\end{array}$ \\
Q5 & $\begin{array}{l}\text { Did you feel that the peer agent cared } \\
\text { about you? (Agent's caring) }\end{array}$ \\
Q6 & $\begin{array}{l}\text { Was the peer agent likable? (Agent's lik- } \\
\text { ability) }\end{array}$ \\
Q7 & $\begin{array}{l}\text { Did the peer agent support you? } \\
\text { (Agent's support) }\end{array}$ \\
\hline
\end{tabular}

Table 3: Questionnaire items to evaluate user satisfaction (Q1, Q2, and Q3) and user opinions about the peer agent $(\mathrm{Q} 4, \mathrm{Q} 5, \mathrm{Q} 6$, and Q7)

user utterances. Among these measures, we regarded the number of user utterances as an objective measure to evaluate communication activation. User satisfaction and opinions about the peer agent are subjective measures based on the questionnaires (ten-point Likert scale). Table 3 shows the questionnaires used in the experiment. We expected that a high level of user satisfaction and positive opinions about the peer agent would lead to a high level of user engagement, which would promote user utterances.

User satisfaction was evaluated from different perspectives with three questions: Q1, Q2, and Q3. Q1 focused on user willingness to engage in the dialogue; Q2 focused on the user experience of the dialogue's pleasantness; Q3 focused on user satisfaction with the system. We evaluated user satisfaction with averages of the ratings of Q1, Q2, and Q3. Using the averaged ratings of Likert questions allows us to apply such parametric statistical tests as a multi-factor ANOVA since the summed or averaged responses to Likert questions tend to follow a normal distribution.

User opinions about the peer agent were evaluated in terms of how the user perceived the peer agent's closeness (Q4), its caring (Q5), its likability (Q6), and its support (Q7). We evaluated user opinions about the peer agent with the averaged ratings of these items. Previous studies showed that empathic behaviors exhibited by an agent im- proved user opinions about the agent in a Blackjack scenario (Brave et al., 2005) and in a social dialogue between a single user and an agent (Higashinaka et al., 2008). We examined these items in multi-party dialogues with flexible turn-taking.

\subsection{Procedure}

We recruited and paid 64 Japanese adults (32 males and 32 females) for their participation. The mean ages of the male and female groups were 32.0 and 36.2, respectively (male group: $\mathrm{SD}=9.2$ , $\min =22, \max =59$, female group: $\mathrm{SD}=9.6$, $\min =20, \max =50$ ). The participants were divided into 32 pairs of the same gender: 16 pairs of males and 16 pairs of females. The participants in each pair were unacquainted.

The experiment had a within-participants design. Each pair of participants successively engaged in dialogues using the five systems under different experimental conditions. The order of using the systems was counter-balanced to prevent order effect.

Before starting the experiment, the participants were informed that, after completing a dialogue with each system, they would fill out questionnaires. The questionnaires on user opinions about the peer agent were used only when it was present (conditions (1), (2), (3), and (4)). The participants were also told that the agents were computer programs and not human participants. During the experiment, each pair of participants was seated in separate rooms in front of a computer display, a keyboard, and a mouse, and they could only communicate with each other through the system.

In the dialogue with each system, five "Who is this?" quizzes about famous people were presented. The quiz subjects were chosen so that the difficulty level of the quizzes was approximately the same in all the systems. For this purpose, we first sorted people in Wikipedia in descending order by their PageRank ${ }^{\mathrm{TM}}$ score based on Wikipedia's hyper-link structure. We then extracted the top-50 people and divided them from the top into five groups of 10 . Next we randomly selected five people from each group to make five sets of five people of approximately identical PageRank scores. Each set of five people was used for quizzes in each system.

On average, a pair of participants took $18 \mathrm{~min}$ utes to complete a dialogue with each system. The number of hints that were actually presented in a 


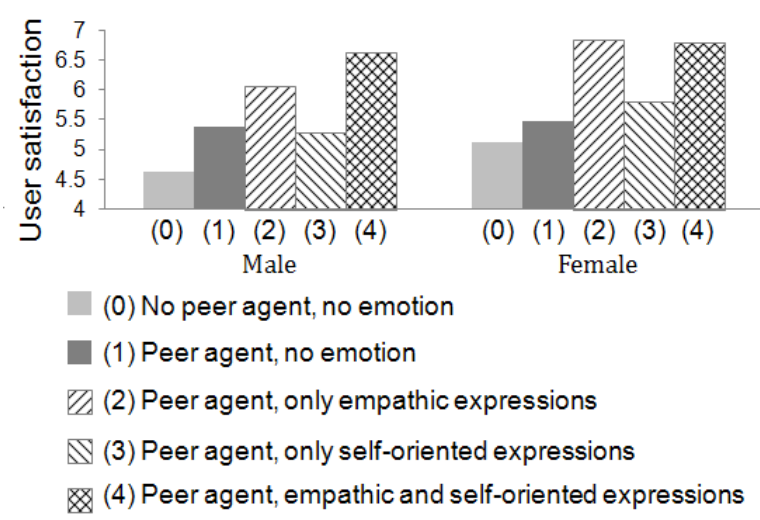

Figure 2: User satisfaction

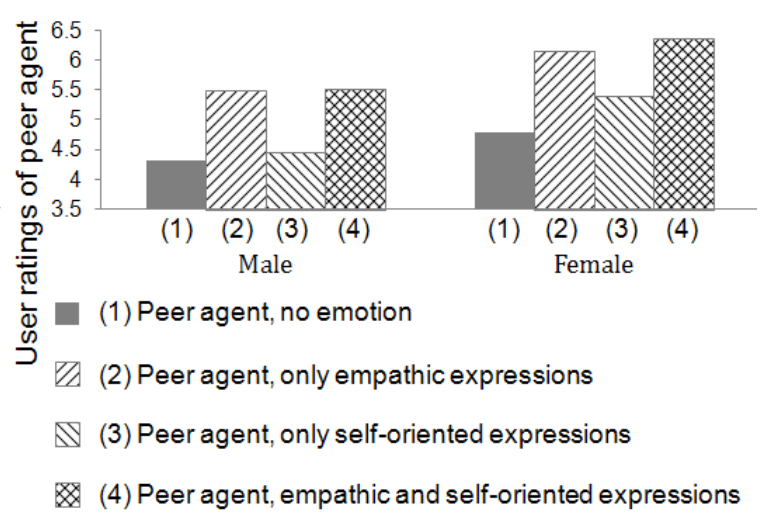

Figure 3: User ratings of peer agent

These results showed that the peer agent's presence and the agent empathic expressions significantly improved user satisfaction in quiz-style multi-party dialogues.

\subsection{User opinions about the peer agent}

For questions Q4, Q5, Q6, and Q7, Cronbach's alpha was 0.92 , which justified combining these items into a single index. Therefore we evaluated user opinions about the peer agent with the averaged ratings of these items under each experimental condition. Figure 3 shows the user ratings of the peer agent under each condition.

To evaluate the effect of agent empathic and self-oriented expressions on the user ratings of the peer agent, we compared conditions (1), (2), (3) and (4). A three-factor ANOVA was conducted with two within-participant factors of empathic and self-oriented expressions and one betweenparticipant factor of gender. The F-test for the homogeneity of variances $(p>0.3)$ and the Kolmogorov-Smirnov normality test $(p>0.2)$ showed that the data met the ANOVA assumptions. As a result of the ANOVA, a significant main effect was found for empathic expressions with respect to the user ratings of the peer agent, $F(1,62)=77.4, p<0.001$. There was a moderate main effect for self-oriented expressions with respect to the user ratings of the peer agent, $F(1,62)=4.38, p<0.04$. There were no significant main effects for gender, and there were no significant interactions.

These results showed that agent empathic expressions significantly improved user ratings of the peer agent in quiz-style multi-party dialogues. 


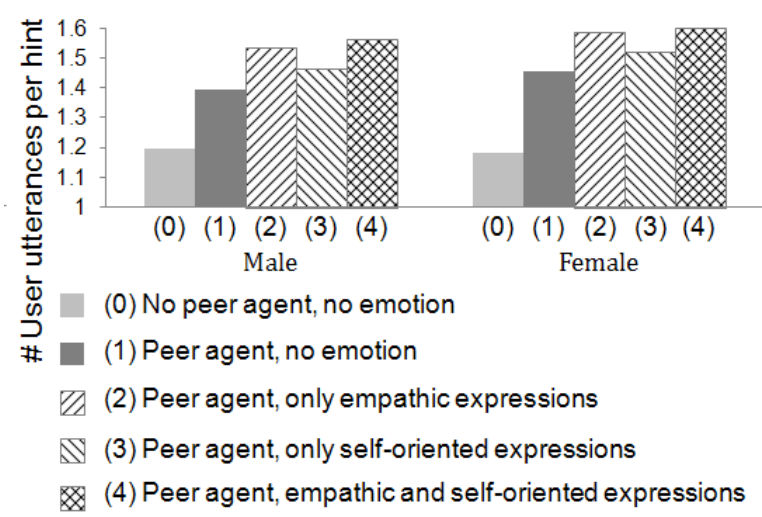

Figure 4: User utterances per quiz hint

\subsection{Number of user utterances}

Figure 4 shows the number of user utterances per quiz hint under each condition.

To evaluate the effect of the peer agent's presence on the number of user utterances per quiz hint, we compared conditions (0) and (1). Based on the F-test and the Kolmogorov-Smirnov test, the assumptions of variance homogeneity $(p>$ $0.6)$ and normality $(p>0.5)$ were met. By applying the paired t-test to both the male and female groups, we found that the presence of the peer agent significantly increased the number of user utterances per hint (male group: $t(31)=3.1, p<$ 0.004 , female group: $t(31)=5.6, p<0.001)$.

To evaluate the effect of empathic and selforiented expressions by agents on the number of user utterances, we compared conditions (1), (2), (3), and (4). A three-factor ANOVA was conducted with two within-participant factors of empathic and self-oriented expressions and one between-participant factor of gender. The F-test for the homogeneity of variances $(p>0.05)$ and the Kolmogorov-Smirnov normality test $(p>0.6)$ showed that the data met the ANOVA assumptions. As a result of the ANOVA, a significant main effect was found for empathic expressions with respect to the number of user utterances, $F(1,62)=18.9, p<0.001$. No significant main effects were found for either self-oriented expressions or gender, and there were no significant interactions.

These results showed that the peer agent's presence and agent empathic expressions increased the number of user utterances and stimulated human communication in quiz-style multi-party dialogues.

\section{Conclusion}

This paper experimentally analyzed how conversational agents stimulate human communication in thought-evoking multi-party dialogues between multi-users and multi-agents. As an example of such multi-party dialogue, we focused on quizstyle multi-party dialogues between two users and two agents. We investigated how a peer agent's presence and agent emotional expressions influenced user satisfaction, the user ratings of the peer agent, and the number of user utterances. The user ratings of the peer agent included user's perceived closeness, likability and caring from the peer agent, and the user's feeling of being supported by the peer agent.

The experiment results showed that the peer agent's presence significantly improved user satisfaction and increased the number of user utterances. We also found significant effects that agent empathic expressions improved user satisfaction and user positive ratings of the peer agent and that they further increased the number of user utterances. These results indicate that employing a peer agent and agent empathic behaviors in thoughtevoking multi-party dialogues will stimulate interaction among people in computer-mediated communication. Our findings will be useful for a broader class of applications such as educational agents and community facilitators.

Many directions for future work remain. First, we plan to extend our work to deal with various modalities such as speech, gestures, body posture, facial expressions, and the direction of eye gazes to investigate the effects of agent representation (embodied or disembodied) and other modalities in thought-evoking multi-party dialogues. Second, we will analyze how agent behaviors influence users and dialogues in more detail and develop a more sophisticated dialogue management method based on our detailed analysis. Learning optimal dialogue management strategies in multi-party dialogues is a challenging research topic. Third, examining the relationship between user personality traits and the impact of agents on users is valuable. Previous work reported that the effect of embodiment depended on user personalities (Lee et al., 2006). This direction is important to the stimulation of multi-party interaction for therapeutic and emotional support. 


\section{References}

James Allen, Donna Byron, Myroslava Dzikovska, George Ferguson, Lucian Galescu, and Amanda Stent. 2001. Toward conversational humancomputer interaction. AI Magazine, 22(4):27-37.

Joseph Bates. 1994. The role of emotion in believable agents. Communications of the ACM, 37(7):122125 .

Timothy W. Bickmore and Rosalind W. Picard. 2005. Establishing and maintaining long-term humancomputer relationships. ACM Transactions on Computer-Human Interaction, 12(2):293-327.

Scott Brave, Clifford Nass, and Kevin Hutchinson. 2005. Computers that care: investigating the effects of orientation of emotion exhibited by an embodied computer agent. International Journal of HumanComputer Studies, 62(2):161-178.

Justine Cassell, Joseph Sullivan, Scott Prevost, and Elizabeth Churchill, editors. 2000. Embodied Conversational Agents. MIT Press, Cambridge, MA.

Chih-Yueh Chou, Tak-Wai Chan, and Chi-Jen Lin. 2003. Redefining the learning companion: the past, present, and future of educational agents. Computers \& Education, 40(3):255-269.

Scotty D. Craig, Barry Gholson, Matthew Ventura, Arthur C. Graesser, and the Tutoring Research Group. 2000. Overhearing dialogues and monologues in virtual tutoring sessions: Effects on questioning and vicarious learning. International Journal of Artificial Intelligence in Education, 11:242253.

Patrick Gebhard, Martin Klesen, and Thomas Rist. 2004. Coloring multi-character conversations through the expression of emotions. In Lecture Notes in Computer Science (Tutorial and Research Workshop on Affective Dialogue Systems), volume 3068, pages 128-141.

Ryuichiro Higashinaka, Kohji Dohsaka, Shigeaki Amano, and Hideki Isozaki. 2007a. Effects of quizstyle information presentation on user understanding. In Proceedings of the 8th Annual Conference of the International Speech Communication Association, pages 2725-2728.

Ryuichiro Higashinaka, Kohji Dohsaka, and Hideki Isozaki. 2007b. Learning to rank definitions to generate quizzes for interactive information presentation. In Proceedings of the 45th Annual Meeting of the Association for Computational Linguistics (Poster Presentation), pages 117-120.

Ryuichiro Higashinaka, Kohji Dohsaka, and Hideki Isozaki. 2008. Effects of self-disclosure and empathy in human-computer dialogue. In Proceedings of 2008 IEEE Workshop on Spoken Language Technology, pages 109-112.
Eva Hudlicka. 2003. To feel or not to feel: The role of affect in human-computer interaction. International Journal of Human-Computer Studies, 59(1-2):1-32.

Kwan Min Lee, Younbo Jung, Jaywoo Kim, and Sang Ryong Kim. 2006. Are physically embodied social agents better than disembodied social agents?: Effects of embodiment, tactile interaction, and people's loneliness in human-robot interaction. International Journal of Human-Computer Studies, 64(10):962-973.

Yi Liu and Yam San Chee. 2004. Intelligent pedagogical agents with multiparty interaction support. In Proceedings of International Conference on Intelligent Agent Technology, pages 134-140.

Heidy Maldonado, Jong-Eun Roselyn Lee, Scott Brave, Cliff Nass, Hiroshi Nakajima, Ryota Yamada, Kimihiko Iwamura, and Yasunori Morishima. 2005. We learn better together: enhancing elearning with emotional characters. In Proceedings of the 2005 Conference on Computer Support for Collaborative Learning, pages 408-417.

Rosalind W. Picard. 1997. Affective Computing. MIT Press, Cambridge, MA.

Helmut Prendinger and Mitsuru Ishizuka, editors. 2004. Life-Like Characters: Tools, Affective Functions, and Applications. Springer, Berlin.

Helmut Prendinger, Junichiro Mori, and Mitsuru Ishizuka. 2005. Using human physiology to evaluate subtle expressivity of a virtual quizmaster in a mathematical game. International Journal of Human-Computer Studies, 62(2):231-245.

Keith Stenning, Jean McKendree, John Lee, Richard Cox, Finbar Dineen, and Terry Mayes. 1999. Vicarious learning from educational dialogue. In Proceedings of the 1999 Conference on Computer Support for Collaborative Learning, pages 341-347.

David Traum and Jeff Rickel. 2002. Embodied agents for multi-party dialogue in immersive virtual worlds. In Proceedings of the 1st International Joint Conference on. Autonomous Agents and Multi-Agent Systems, pages 766-773.

Jun Zheng, Xiang Yuan, and Yam San Chee. 2005. Designing multiparty interaction support in Elva, an embodied tour guide. In Proceedings of the 4th International Joint Conference on Autonomous Agents and Multiagent Systems, pages 929-936.

Victor Zue, Stephanie Seneff, Joseph Polifroni, Michael Phillips, Christine Pao, David Goodine, David Goddeau, and James Glass. 1994. PEGASUS: a spoken dialogue interface for on-line air travel planning. Speech Communication, 15:331340 . 